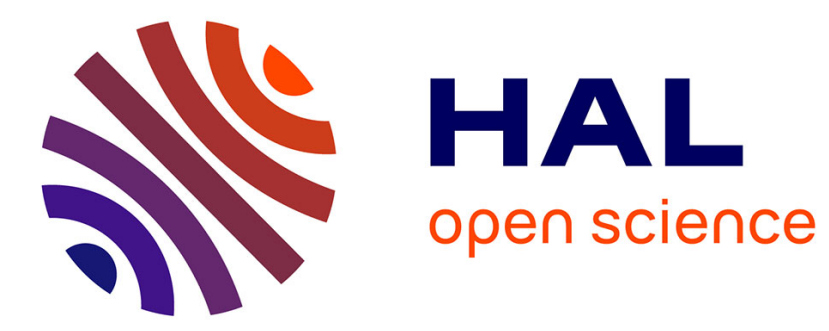

\title{
De la terreur au terrorisme: les aventures d'une philosophie de l'histoire chez Merleau-Ponty
}

Thamy Ayouch

\section{To cite this version:}

Thamy Ayouch. De la terreur au terrorisme: les aventures d'une philosophie de l'histoire chez Merleau-

Ponty. Topique - Revue freudienne, 2002, 81, pp.125-140. 10.3917/top.081.0125 . halshs-00940615

\section{HAL Id: halshs-00940615 \\ https://shs.hal.science/halshs-00940615}

Submitted on 8 Feb 2014

HAL is a multi-disciplinary open access archive for the deposit and dissemination of scientific research documents, whether they are published or not. The documents may come from teaching and research institutions in France or abroad, or from public or private research centers.
L'archive ouverte pluridisciplinaire HAL, est destinée au dépôt et à la diffusion de documents scientifiques de niveau recherche, publiés ou non, émanant des établissements d'enseignement et de recherche français ou étrangers, des laboratoires publics ou privés. 


\title{
DE LA TERREUR AU TERRORISME : LES AVENTURES D'UNE PHILOSOPHIE DE L'HISTOIRE CHEZ MERLEAU-PONTY ?
}

\author{
Thamy Ayouch
}

\section{L'Esprit du temps | Topique}

\author{
$2002 / 4$ - no 81 \\ pages 125 à 140
}

ISSN 0040-9375

Article disponible en ligne à l'adresse:

http://www.cairn.info/revue-topique-2002-4-page-125.htm

Pour citer cet article

Ayouch Thamy, « De la terreur au terrorisme : les aventures d'une philosophie de l'histoire chez Merleau-Ponty ? », Topique, $2002 / 4$ no 81 , p. 125-140. DOI : 10.3917/top.081.0125

Distribution électronique Cairn.info pour L'Esprit du temps.

(C) L'Esprit du temps. Tous droits réservés pour tous pays.

La reproduction ou représentation de cet article, notamment par photocopie, n'est autorisée que dans les limites des conditions générales d'utilisation du site ou, le cas échéant, des conditions générales de la licence souscrite par votre établissement. Toute autre reproduction ou représentation, en tout ou partie, sous quelque forme et de quelque manière que ce soit, est interdite sauf accord préalable et écrit de l'éditeur, en dehors des cas prévus par la législation en vigueur en France. II est précisé que son stockage dans une base de données est également interdit. 


\title{
De la terreur au terrorisme: les aventures d'une philosophie de l'histoire chez Merleau-Ponty?
}

\author{
Thamy Ayouch
}

«Une société n'est pas le temple des valeurs idoles qui figurent au fronton de ses monuments ou dans ses textes constitutionnels, elle vaut ce que valent en elle les relations de l'homme avec l'homme».

Maurice Merleau-Ponty, Humanisme et terreur

Le terrorisme le plus souvent évoqué actuellement est le fait d'individus ou d'organisations, ce qui fait parfois oublier l'origine étatique du vocable «terrorisme». La Terreur désigne les mesures d'exceptions prises par le gouvernement révolutionnaire depuis la chute des Girondins (en juin 1793) jusqu'à celle de Robespierre (le 27 juillet 1794). Terreur et terrorisme ne se confondent toutefois pas. En creusant ici la différence sémantique entre les deux nous aboutirons à une première spécification du terrorisme d'État. La terreur, d'abord passion au $\mathrm{XVII}^{\mathrm{e}}$ siècle, acquiert seulement au XVIII ${ }^{\mathrm{e}}$ siècle un sens collectif qui la lie à une finalité dans la double dimension de son destinataire et de son objectif. Elle frappe quelqu'un, individu ou groupe, pour faire obtenir quelque chose. Le terme «terrorisme » connaît une dérive sémantique inverse : du collectif étatique au particulier. S'il est appliqué d'abord au gouvernement institué par la Convention lorsque celui-ci est condamné, il désigne par la suite un élément d'intimidation, voire d'épouvante, qui joue de l'art de la menace et des craintes de l'adversaire. On distingue habituellement ici deux réalités différentes : d'une part les actes violents que perpètrent des individus isolés ou des organisations 
dans le but de lutter contre les appareils d'État - et nous les nommerons terrorisme d'individus ou d'organisation ; d'autre part, le régime d'oppression et de répression utilisé par un gouvernement pour se maintenir au pouvoir, terrorisme institutionnalisé ou terrorisme d'État. Outre la considération des agents, la distinction de ces deux types de terrorisme repose sur les temporalités différenciées qu'ils mettent en œuvre. Irruption brutale du hasard, l'acte terroriste d'un individu ou d'une organisation s'inscrit dans l'immédiateté, a pour finalité l'instantanéité d'une réaction, se fonde sur une logique des effets dans sa recherche volontaire de ces réactions, et se définit par son événementialité. Le terrorisme d'État, au contraire, déploie une temporalité plus longue et vise non pas l'instantanéité d'une réaction, mais la pérennité d'une domination idéologique éradiquant toute pensée dissidente et toute opposition politique. C'est davantage à une logique des causes qu'il obéit, en recherchant ce qui légitime, justifie, fonde et nourrit la terreur. En outre, dans le terrorisme d'organisation, l'événement violent est ce qui crée la relation terroriste et construit un conflit (entre l'organisation et l'autorité ou l'État), conflit qui disparaît sitôt que l'organisation est démantelée, et qui, pour exister, doit être nourri par des actions spectaculaires. Il se joue donc devant les médias qui garantissent la transmission d'une proximité de la terreur. Le terrorisme d'État, lui, par une gestion bureaucratique de la répression, n'entend point créer le conflit, mais plutôt le nier. Par des procédures morales, intellectuelles, physiques d'exclusion ou de violence, il tente de perpétuer un ordre politique en annulant toute logique conflictuelle, toute expression de l'opposition et toute procédure de négociation. À l'opposé $\mathrm{du}$ terrorisme d'organisation qui conçoit son acte comme injection d'un désordre, le terrorisme d'État développe une logique de la peur au service de l'ordre et y emploie des moyens chaque fois plus irraisonnés, puisque nécessairement impuissants à assurer un ordre total. C'est là ce qui en fait un totalitarisme.

Nous utiliserons, dans la tradition arendtienne, le concept de totalitarisme pour parler de la période stalinienne, malgré toutes les réticences et corrections théoriques dont il a fait l'objet. Le terme, apparu d'abord dans l'apologie que Giovanni Gentile fait du fascisme, présente un idéal politique où seul le tout social a une valeur pour lui-même, et les individus ne sont que des organes à son service. Pour Hannah Arendt, il désigne une catégorie politique d'un type radicalement nouveau, où un parti unique, seul détenteur du sens de l'Histoire, dispose d'un contrôle absolu qui s'exerce non pas sur une société constituée mais sur des masses atomisées (H. Arendt, Le Système totalitaire (Les Origines du totalitarisme, tome 3 ). Mais ce qui nous pousse davantage à nous situer dans la perspective de H. Arendt est qu'elle caractérise l'État totalitaire par le niveau de terreur policière, physique et psychologique sans précédent qu'il met en place. Cette terreur, dont le but est d'éliminer systématiquement tous les opposants, investit complètement la vie privée des individus par un contrôle idéologique destiné à remodeler les hommes et à consolider le pouvoir. La différence entre État et société civile, espaces public et privé disparaît, entraînant 
un système d'équivalences $:$ société $=$ État $=$ Parti $=$ guide charismatique . Les camps de concentration montrent alors comment le processus de terreur mis en place et visant l'élimination de tout opposant (des peuples ou classes entières considérés comme ennemis objectifs) en arrive à la poursuite aveugle d'un raisonnement logique qui n'est plus soutenu par le sens commun. C'est ici qu'apparaît le plus clairement le terrorisme d'État. Là où la terreur est inséparable d'une finalité qu'elle commence par placer hors d'elle, le terrorisme d'État établit une logique de la peur au service de toujours plus d'ordre, finit par ne plus viser que lui-même, et devient terreur totale, totalitarisme.

La distinction entre terreur et terrorisme d'État s'avère donc d'envergure. Si un régime est taxé de terrorisme d'État, instrument d'oppression conduit à toujours plus de violence car voulant toujours plus d'ordre, il est parfaitement condamnable. Si en revanche, on ne parle que de la terreur qu'il met en œuvre, inséparable d'une finalité autre qu'elle-même, il apparaît comme mouvement de révolte contre un ordre établi qu'il souhaite renverser, retrouve, dans le cas de la Première République française ou du régime soviétique, sa filiation révolutionnaire, et n'est pas instrument d'oppression, mais lutte contre l'oppression.

C'est cette distinction qui fonde implicitement l'analyse qu'effectue Merleau-Ponty de la terreur dans le régime soviétique. La question centrale que le philosophe traite tout le long de Humanisme et Terreur est de savoir si la terreur mise en œuvre par ce régime est susceptible de se dépasser vers un humanisme. Il s'agit pour Merleau-Ponty dans ses écrits sur le marxisme (d'abord Humanisme et terreur, puis Les Aventures de la dialectique, et entre temps d'autres textes regroupés dans Sens et non-sens, Signes, ou Parcours) de réfléchir sur le processus de transformation de la terreur en terrorisme d'État. Il semble ici pertinent de présenter l'analyse que Merleau-Ponty effectue de la terreur, pour voir comment, aboutissant au terrorisme d'État, il est conduit à réviser les outils théoriques et pratiques dont il dispose pour comprendre l'histoire et s'y positionner. Lorsque, choisissant une perspective philosophique et herméneutique pour rendre compte de la terreur, Merleau-Ponty est amené, devant le terrorisme d'État avéré, à la critique de cette perspective, c'est la question de la possibilité même d'une compréhension du terrorisme par une théorisation issue des sciences humaines qui se trouve posée.

\section{LA TERREUR, UNE VIOLENCE QUI SE DÉPASSE?}

La réflexion de Merleau-Ponty sur la terreur et le terrorisme ne peut être détachée de son analyse générale de la violence. Le contexte historique de formation progressive de deux blocs ennemis après la deuxième guerre mondiale favorise chez le philosophe la thèse d'une violence inhérente à tout type de régime, inscrite dans l'histoire même, et dont le déni ou l'ignorance relèverait d'une morale hypocrite. La morale libérale, qui prétend enseigner la nonviolence, consolide la violence établie, identifiée par Merleau-Ponty dans la 
préface de Humanisme et terreur comme système de production qui rend inévitables la misère et la guerre; et l'humanisme occidental apparent ne serait qu'un autre usage de la violence. Marquant dès 1945 sa distance envers tout système politique, il renvoie dos à dos l'anticommuniste qui refuse de voir que la violence est partout, et le sympathisant communiste exalté qui tendrait à en réduire l'horreur. C'est là le sens de son propos dans sa «Note sur Machiavel» (in Éloge de la philosophie) où, soulignant l'idée machiavélienne du pouvoir toujours contestable et menacé, il identifie l'agression que le pouvoir écarte du lui et celle qu'il renvoie. C'est seulement en reconnaissant la présence de cette violence que peut être abordée la question d'un pouvoir qui ne soit pas injuste, et qu'à la différence de Machiavel Marx pose.

En replaçant les Procès de Moscou dans la Stimmung révolutionnaire de la violence, la question alors introduite n'est pas de savoir si le communisme respecte la pensée libérale, et il ne le fait pas, mais si sa violence est révolutionnaire et entend créer de nouveaux rapports humains. Le marxisme, accordé aux faits de l'histoire qu'il entend déchiffrer, se place à égale distance d'une philosophie visionnaire qui déciderait d'un sens a priori, et d'un terrorisme sans perspectives. La violence qu'il pourrait légitimer, en donnant de la situation historique du libéralisme un tableau pessimiste dès le départ, doit donc porter en elle-même le signe d'autre chose.

La violence est liée par Merleau-Ponty au rôle de la contingence dans l'histoire. Parce que l'histoire n'est pas le développement d'une raison prédéterminée, mais l'effectuation d'un sens dans les rapports entre les hommes, parce que la rencontre de leurs projets individuels reste imprévisible, le sens de leurs actes transcendera toujours leurs intentions. La responsabilité n'est pas alors ce que les hommes ont voulu, mais ce qu'ils se trouvent avoir fait à la lumière de l'événement. Si «la malédiction de la politique tient justement en ceci qu'elle doit traduire des valeurs dans l'ordre des faits » (Humanisme et terreur, p. 64), le tragique du politicien est cette discrépance entre ses intentions et ses actes, le drame d'une honnêteté subjective et d'une trahison objective, contradictions que Merleau-Ponty lit chez Boukharine dans les Procès de Moscou. Ces derniers rendent leurs verdicts au nom d'une vérité que la Révolution est encore en train d'effectuer, ne se préoccupent ni des mobiles ni des intentions des accusés, mais des résultats de leurs actions dans la perspective d'une histoire universelle, pourtant inachevée. Ils ne sont alors compréhensibles qu'entre révolutionnaires, à savoir entre hommes convaincus de faire l'histoire. Toutefois, et c'est là que Merleau-Ponty dénonce les méthodes du régime, ce sont des procès révolutionnaires présentés comme des procès ordinaires où les opposants sont maquillés en espions ou saboteurs. Quand l'existence du régime est remise en question, la lutte politique ne se distingue plus de ma sphère pénale, l'erreur politique de la trahison, l'humanisme est suspendu et la terreur gouverne. Le danger ici pour Merleau-Ponty est que soit mis «sur la justice révolutionnaire le masque du code pénal» (Humanisme et terreur, p. 122) et que la violence ainsi cachée soit rendue institutionnelle. 
On contrevient toutefois à la seule contingence de l'histoire en lui associant, dans la résolution des hommes, une interprétation, l'investissement d'un sens choisi car décidant de lire dans le présent l'esquisse d'un avenir particulier.

C'est au nom de la vérité que la terreur révolutionnaire est exercée. Toutefois, si la vérité, déchiffrement de la conjoncture et élaboration de la ligne politique, est l'œuvre des théoriciens du Parti, elle ne peut, en bonne logique marxiste, être imposée aux prolétaires contre leur accord: son désaveu signifie qu'elle n'est pas vérité. La violence ne peut alors se prévaloir d'un absolu du vrai : elle n'est nécessaire que parce qu'il n'y a pas de vérité dernière du monde contemplé, et la Stimmung du marxisme est de «n'être pas dans la vérité, mais au seuil de la vérité, qui est à la fois toute proche, indiquée par tout le passé et tout le présent, et à la distance infinie d'un avenir qui est à faire » (Les Aventures de la dialectique, p. 73).

Merleau-Ponty entretient un constant débat avec lui-même et ne tranche pas encore dans Humanisme et terreur: d'un côté, la violence révolutionnaire devrait être préférée pour son avenir humaniste, de l'autre, la violence du présent, toute révolutionnaire qu'elle soit, ne se distingue pas des autres violences. C'est là le paradoxe d'une terreur vingt ans après la Révolution de 1917, où le rapport entre présent et futur est occulté, et l'importance du prolétariat diminuée par le régime. Le bilan en 1947 accuse l'écart entre l'URSS et le modèle marxiste: de nouvelles différenciations sociales apparaissent, la dictature se retrouve renforcée, les procès politiques présentés comme des procès de droit commun et tranchés par une véritable mascarade de jugement, et la Révolution, devenue entreprise toute volontaire, semble perdre son sens. À la question de savoir si la terreur est à même de se dépasser en humanisme, la réponse est négative. Néanmoins, dans Humanisme et terreur est conservée cette foi dans la philosophie de l'histoire marxiste, parce que le prolétariat existe toujours, mais aussi parce que le discrédit du marxisme comme philosophie de 1'histoire signifierait alors pour Merleau-Ponty la perte de tout sens, de tout humanisme, et de toute raison dans l'histoire. Ce n'est qu'en 1950, dans «L'URSS et les camps » (in Signes) que Merleau-Ponty, confronté au Code du travail correctif soviétique présenté à l'ONU, écrit qu' «il n'y a pas de socialisme quand un citoyen sur vingt est aux camps». Il prend alors acte de la déportation légalisée ou de l'appareil répressif organisé comme d'une remise en question totale de la signification du régime russe. Ce constat d'un véritable terrorisme d'État, du fait de la différence de plus en plus creusée entre communisme et marxisme, conduit Merleau-Ponty à s'interroger sur l'outil même qui en permet l'analyse: la dialectique marxiste.

\section{LA CRISE DE LA DIALECTIQUE}

Le concept de dialectique est constant pour Merleau-Ponty tout le long de son œuvre, depuis La Structure du comportement, où il aborde le fonction- 
nement dialectique de la structure (physique, vitale ou humaine) et de la conscience, jusqu'au Visible et l'invisible, où la vie est mouvement dans lequel se nouent l'être et l'expérience de l'être. La dialectique advient à deux niveaux dans la pensée de Merleau-Ponty : elle est mouvement d'une pensée exprimant l'appartenance réciproque et le passage l'un dans l'autre de termes que l'entendement oppose (sujet/objet, positif/négatif, fini/infini); mais elle est aussi mouvement de l'être et du réel, et passage l'un dans l'autre de ces moments opposés. Cette prévalence de la dialectique explique une "phénoménologisation» des pensées hégélienne et marxiste : Merleau-Ponty commence par voir en chacun de ces philosophes une perspective existentialiste (comme le montrent des textes tels que «L'existentialisme chez Hegel» ou «Marxisme et philosophie », in Sens et non-sens), pour dénoncer, par la suite, le scientisme que chacun développe et qui a raison de sa dialectique antérieure. Dans l'histoire, la dialectique est toute marxiste : elle consiste en ce renvoi entre sujet et objet, conscience et histoire, présent et avenir, jugement et discipline, réel et valeurs.

L'alternative que posent A. Koestler dans son roman Darkness at Noon, et le régime communiste dans sa pratique, entre le commissaire - agissant du dehors et traitant les hommes comme des instruments - et le yogi - contenu tout entier dans sa pensée intérieure - est lue par Merleau-Ponty comme un contresens de la dialectique marxiste. Une disjonction de la dialectique a lieu lors des Procès de Moscou, où les actions des accusés ne sont jugées par le procureur que dans la perspective objective d'un sens de l'histoire non pas seulement indiqué dans le présent mais écrit de toute éternité. Une lecture du marxisme y découvrant des chemins historiques objectifs et nécessaires déjà tracés est erronée, puisque le marxisme, comme praxis, crée le sens plutôt qu'il ne le découvre déjà fait.

Si une philosophie marxiste de l'histoire aborde comme indissociables les trois thèmes fondamentaux de l'initiative des masses, de l'internationalisme, et de la construction des bases économiques, force est d'admettre que la situation de l'URSS lors de la Révolution et vingt ans après n'en permet pas la réalisation conjointe. La Révolution reste nationale, et les masses sont écartées de tout pouvoir décisionnel que le Parti assume seul. Le thème du développement économique passe donc au premier plan, aux dépens des deux autres qui restent relégués au futur. Le facteur objectif des bases économiques est alors surestimé, et le facteur subjectif de la conscience prolétarienne sous-estimé. La révolution n'est pas tant le fait de la croissance du prolétariat national ou mondial, qu'une entreprise purement volontaire du Parti. La dialectique n'est plus alors théorie et praxis dans la société soviétique de 1947, elle se réduit à un positionnement extra-historique qui se prévaut de la connaissance d'une histoire universelle.

Se pose alors la question de savoir si cette rupture de la dialectique qui précipite le passage de la terreur au terrorisme d'État est le fait de sa lecture communiste, ou réside déjà dans la philosophie marxiste elle-même. Dans Humanisme et terreur, Merleau-Ponty refuse de discréditer le marxisme devant 
l'échec de son application en URSS. Le déclin de l'humanisme prolétarien n'annule pas le marxisme, qui vaut toujours comme critique du monde existant, et du pseudo-humanisme libéral. Et cette position, déjà développée dans «Autour du marxisme» (in Sens et non-sens) est reprise dans d'autres textes tel «Le Manifeste communiste a cent ans» (in Parcours I). Ce n'est que dans Les Aventures de la dialectique que le marxisme comme philosophie permettant de situer les événements, et en l'occurrence le terrorisme, dans une perspective historique, est attaqué en lui-même. Ce désaveu de la dialectique marxiste réside dans l'idée qu'elle prépare dans sa théorisation même l'avènement d'un réel terrorisme d'État sitôt que la Révolution s'institue au pouvoir.

En montrant comment Lénine, dans Matérialisme et empirocriticisme, installe la dialectique dans les choses, Merleau-Ponty souligne le dogmatisme de cette théorisation du marxisme. Ce dogmatisme est lié à trois points: le scientisme d'un marxisme positiviste, la gnoséologie d'un sujet hors du tissu de l'histoire, et la disparition de l'autocritique. Lénine, selon Merleau-Ponty, reviendrait à un Hegel non existentialiste, ou à un Marx scientiste postérieur au Marx de la dialectique. Deux marxismes apparaissent alors : 1'un, philosophique et dialectique, serait applicable aux périodes d'essor de l'histoire ; l'autre, fondé sur le mécanisme d'un processus historique objectif, serait convoqué lors des périodes d'affaissement telle celle que connaît l'URSS. S'il manque au jeune Marx le moyen de traduire l'inertie des infrastructures et l'enlisement des rapports personnels dans les choses, le Marx tardif, lui, détruit la dialectique en dissociant le subjectif de l'objectif. Juxtaposition de ces deux perspectives, le marxisme est donc confronté au dilemme de ne pas exister comme théorie complète, ou de figer la dialectique. De même, selon MerleauPonty, la gnoséologie de Lénine annule la dialectique en la fondant dans l'être ou dans l'objet pur, en remplaçant l'histoire par une seconde nature opaque et toute déterministe. L'appareil du Parti concentre alors tout le mouvement de l'histoire et du savoir, et réclame une adhésion massive à un processus social - naturaliste - tout entier contenu en lui. La pensée est jugée à l'étalon de sa conformité aux thèses du Parti, dans ce que nous pourrions nommer ici un véritable terrorisme de pensée. Enfin, le marxisme du Parti ne s'applique plus à lui-même: l'autocritique est rendue caduque. L'orthodoxie réclame la vérité des choses mêmes, elle n'accepte pas d'être fondée par la dialectique, qu'elle annule comme instrument critique et ne conserve que comme idéologie. Relisant les Procès de Moscou, Merleau-Ponty y voit le mouvement d'une révolution qui ne veut plus être révolution, d'une pensée communiste qui s'installe dans l'être, hors de la contestation, et devient dogme. La critique n'est plus autocritique, les jugent se confèrent le pouvoir le plus positif, et chargent l'accusé de prononcer la sentence qu'ils portent sur lui.

C'est en outre le rapport même entre Parti et prolétariat qui ne sacrifie plus ici à la dialectique. Celle-ci stipule en effet que la réalité n'est pas, mais devient, et se place donc aux antipodes d'un socialisme scientifique qui se présente comme savoir absolu en se prévalant de traduire un processus historique néces- 
saire en soi. Le Parti, disjoint du prolétariat, légitime alors son usage de la violence par un sens de l'histoire donné une fois pour toutes, et qu'il se charge de lire dans les faits. Même s'il se trompe, le mécanisme scientiste de la révolution permanente le ramènera à lui-même. C'est là déclarer son infaillibilité et instituer le terrorisme de son dogme. Si le marxisme veut présenter la Révolution permanente comme négation continuée immanente au mécanisme de l'histoire, force est d'admettre que le Parti qui s'en prévaut, tout révolutionnaire qu'il puisse être, n'est pas une négation. Il existe positivement, et il n'y a de dictature que du positif. Toutes les identifications médiates de la dialectiques sont ainsi présentées par le Parti comme identités réelles : la société est la révolution, le Parti est le prolétariat, les chefs sont le Parti, ce qui rejoint ici les systèmes d'équivalences propres au totalitarisme que nous avons évoqués. La dialectique étant tout entière située dans l'objet, dans la vérité acquise d'une histoire universelle, on répond alors à toute adversité par une terreur exercée au nom de cette vérité.

Merleau-Ponty voit une radicalisation de cette perte de la dialectique chez Sartre. Pour ce dernier, l'histoire n'est plus action et sens, elle est soit résultat immédiat de nos volontés, soit opacité impénétrable. Extrême objectivisme d'une histoire sans sens, où l'action du Parti est soustraite aux critères de sens, extrême subjectivisme d'une action de l'ordre de la création pure, ces philosophies du sujet pur ou de l'objet pur sont également terroristes pour Merleau-Ponty. S'opposer au PC revient inévitablement, selon Sartre, à s'opposer au prolétariat, car le Parti est dépositaire de la conscience pure. Il ne peut donc y avoir de dissension. Mais cette action pure, et ce Parti unanime, sont, pour Merleau-Ponty action et Parti vus de l'extérieur, de la part de l'intellectuel, du «mandarin », théoricien pur qui ne cherche pas tant à comprendre l'histoire qu'à prouver dogmatiquement une théorie de l'histoire. De cette négation de la dialectique, qui ne voit pas la politique prolétarienne dans une interaction entre classe et Parti, résulte un véritable terrorisme : l'action du Parti n'est plus contrebalancée par l'adhésion du prolétariat, contrepoids qui peut seul la garantir du délire historique.

S'il n'y a plus de dialectique, il faut alors, déclare Merleau-Ponty, séculariser le communisme. Mais cette prise d'acte d'un véritable terrorisme d'État qui n'est plus terreur provisoire visant un humanisme, conduit Merleau-Ponty, plus qu'à récuser le communisme, à remettre en question l'instrument même d'analyse qu'est la dialectique marxiste. En effet, se pose ici la question de la possibilité d'une Révolution qui ne se tienne pas elle-même pour absolu, qui puisse faire place à une contestation en elle-même et ne dépouille pas de tout son sens la dialectique. Si la Révolution se conçoit comme fin du gouvernement, elle se pratique comme gouvernement limite, et s'autorise alors de sa propre conception comme abolition du gouvernement pour exercer la terreur. La Révolution n'est prolétarienne qu'avant d'avoir réussi et accédé au pouvoir, et le propre d'une révolution est de se croire absolue et de ne pas l'être justement parce qu'elle le croit. La critique, incarnée historiquement dans une classe qui 
serait suppression de soi, provoque chez ses représentants la conviction d'être l'universel en acte, le droit de s'affirmer sans limite et de pratiquer la terreur au nom d'un humanisme abstrait puisque relégué dans cette dialectique qui n'est plus qu'idéologie. La certitude de parler à la place de l'historie fait de la critique marxiste un dogme et l'empêche d'être autocritique.

\section{ALTÉRITÉ, HERMÉNEUTIQUE ET ENGAGEMENT}

Cette annulation de la dialectique s'accompagne, pour Merleau-Ponty, d'une mise à mal de l'altérité, concept central dans toute sa philosophie. Du marxisme, Merleau-Ponty, choisit d'accentuer la conception de l'intersubjectivité, en montrant que l'humanisme qu'il vise ouvre sur un horizon d'avenir où l'homme est pour l'homme l'être suprême. L'avènement du prolétariat implique la reconnaissance de l'homme par l'homme, et la praxis est avant tout interhumaine. Si pour le révolutionnaire, comme Merleau-Ponty l'indique dans Humanisme et terreur, nous sommes de part en part ce que nous sommes pour autrui et dans nos rapports avec lui, les fondements de la politique marxiste se doivent de mener conjointement une analyse du fonctionnement économique et une intuition des relations interhumaines. Plus encore, les rapports économiques ne sont que l'expression de rapports particuliers entre les hommes. À une simple pluralité de sujets juxtaposés, le dialectique marxiste substitue une réelle intersubjectivité. L'enthousiasme de Merleau-Ponty pour ce traitement de l'intersubjectivité est tel dans Humanisme et terreur, qu'il salue dans le prolétariat la seule intersubjectivité authentique, susceptible de réaliser l'humanité comme relation réciproque entre les hommes.

Lorsque la dialectique est rompue, et le subjectif dissocié de l'objectif, le prolétariat est abandonné, et l'altérité alors en péril. C'est précisément ce qui se passe lorsque la terreur, détachée de tout horizon humaniste, devient terrorisme d'État. Merleau-Ponty dénonce le remplacement par Lénine d'une conception de l'histoire comme relation entre personnes, incarnée dans des choses, par une seconde nature opaque et tout entière mécanique. C'est également là le reproche adressé à Sartre, chez qui une philosophie de la conscience dispose seule de la signification. Il n'y a chez Sartre que des hommes et des choses, et entre les deux des scories de la conscience. De même, il n'y a guère d'autre médiation entre autrui et moi que mon regard, qui me fait sujet et lui objet, ou son regard qui inversement le constitue comme sujet et me constitue pour lui comme objet. De là une violence inhérente aux rapports entre les hommes, héritée d'une radicalisation de la dialectique du maître et de l'esclave de Hegel. Toute l'analyse husserlienne d'apprésentation d'autrui comme alter ego, et de constitution de l'objectivité comme intersubjectivité est ici écartée. Mon rapport à autrui est seulement rapport de conscience à conscience, et l'unique manière pour le prolétaire de franchir l'abîme entre deux consciences est de faire don de lui même au Parti, dans une identification 
totale, laissant ainsi renaître les formes mêmes de société rétrograde que la Révolution voulait dépasser. Mais la socialité ne peut être appréhendée par le seul «je pense» pour qui elle apparaît philosophiquement aporique. À cela, Merleau-Ponty oppose l'incarnation et l'historicité, qui permettent de me lier respectivement au monde, à autrui, et à l'histoire. Si autrui n'est pas seulement constitué par ma conscience, mais existe dans une véritable intersubjectivité fondée sur notre intercorporalité - comme le démontre Merleau-Ponty dans la Phénoménologie de la Perception - je n'agis pas alors envers lui selon le mythe de l'action pure et de la suprême liberté. C'est seulement sur la base d'une intersubjectivité reconnue qu'autrui peut ne pas m'apparaître comme ennemi, menace à ma subjectivité et à ma liberté. La rupture à laquelle aboutit la dialectique dans la Révolution au pouvoir atteint donc cette intersubjectivité fondamentale à mon respect de l'autre, et partant, rend plus indifférent l'exercice de la violence à son endroit. C'est ici une lecture du terrorisme d'État comme radicale négation de l'autre qui est effectuée.

Altérité et sens sont intimement liés chez Merleau-Ponty. Par sens, nous entendons ici le souci de compréhension qui guide toute sa lecture, et lui fait développer une herméneutique historique. Rappelons ici que la problématicité d'une herméneutique historique est liée à la possibilité d'une compréhension de l'histoire comme tout et parties, au sein d'un cercle herméneutique. Le problème posé est celui d'un tout historique qui ne peut jamais être donné, si l'on refuse la perspective d'une histoire de la raison définie a priori (Hegel) ou d'une conscience la surplombant, transcendante à elle. Gadamer, dans Vérité et méthode, recense les différentes réponses à cette aporie, depuis Ranke, jusqu'à Droysen puis Dilthey. Après certains détours par les phénoménologies de Husserl et Heidegger, la possibilité d'une herméneutique historique est trouvée dans l'historicité de l'historien. Toute entière herméneutique, la position de Merleau-Ponty à l'endroit de l'histoire consiste à ne jamais séparer de l'épreuve des événements, vécus ou reconstitués, une philosophie de l'histoire plus englobante. Si la politique est va-et-vient entre le réel et les valeurs, l'attitude de l'historien, pour comprendre cette politique, reproduit ce va-et-vient entre expérience et philosophie. Lorsqu'il pose la question de la compréhension de la terreur, dans Humanisme et terreur, et invite à resituer les Procès de Moscou dans le cadre du marxisme révolutionnaire, il s'agit, non pas de légitimer les faits, mais de permettre qu'un acte prenne sens dans un contexte, qu'un fait n'ait de signification que dans la dynamique d'un régime et dans la totalité historique à laquelle il appartient. C'est donc au nom de la possibilité d'une herméneutique historique que la dialectique marxiste est préconisée comme perspective d'appréhension de l'histoire, et toujours au nom de la possibilité d'une herméneutique historique qu'elle est remise en question. Dans Humanisme et terreur, selon la perspective marxiste, l'histoire se comprend en tant qu'elle se fait, et l'action révolutionnaire identifie philosophie de l'histoire et expérience de l'histoire. De même, dès l'ouverture des Aventures de la dialectique, MerleauPonty réclame une philosophie de l'histoire et de l'esprit qui se construise à 
l'épreuve des événements. Il montre comment Weber souligne l'importance de l'inachèvement de l'histoire, ou de l'union entre philosophie et expérience, et met en exergue la conception qu'a Lukàcs de l'histoire comme philosophie de l'histoire réalisée, et de la philosophie de l'histoire comme histoire formalisée. Dans cette perspective marxiste d'une histoire à la fois vécue, faite et comprise, c'est le prolétariat, premier niveau herméneutique, qui se comprend lui-même et comprend ainsi la société et l'histoire, et c'est l'historien ou philosophe de l'histoire qui ensuite comprend le prolétariat.

Le marxisme scientiste, au contraire, rend l'herméneutique impossible puisqu'il voit la Révolution à l'avance inscrite dans un processus historique donné comme tout transcendant. En intronisant la conscience dans une position extra-historique la faisant surplomber le déroulement des faits, Sartre empêche tout autant une herméneutique historique d'avoir lieu. Le recours à une histoire universelle supposée accomplie n'est ainsi qu'un déguisement du pragmatisme et du nominalisme, destiné à justifier, au nom d'une certitude historique d'humanisme dans le futur, l'usage de la terreur. À considérer l'histoire comme une opacité impénétrable ou comme le résultat immédiat de nos volontés et actions pures, Sartre la soustrait au sens. Il n'y a donc rien à comprendre, il s'agit seulement d'exécuter. Le terrorisme d'État exercé par le Parti se donne alors cette totalité d'une histoire universelle accomplie à laquelle il force l'histoire qui se fait à s'accorder. Ses activités sont confinées à l'absurdité d'un écart qui se creuse entre le sens figé d'une totalité donnée (l'interprétation officielle, qui refuse l'herméneutique d'une constante réinterprétation confrontée au faits) et ce que des éléments divergents proposent comme nouveau sens. Le but du terrorisme d'État sera de réduire cet écart, non pas, comme le fait le mouvement du cercle herméneutique, par un constant retour de la compréhension des parties sur l'interprétation du tout, mais soit en éliminant ces sens différents des parties, soit en supprimant le principe même d'une compréhension.

De ce souci herméneutique résulte un véritable positionnement de l'intellectuel devant l'histoire et le terrorisme. Dans la conclusion de Humanisme et terreur, Merleau-Ponty définit le rôle de l'intellectuel en ces termes: «rendre claire la situation idéologique, souligner, par delà les paradoxes et les contingences de l'histoire présente, les vrais termes du problème humain, rappeler les marxistes à leur inspiration humaniste, rappeler aux démocraties leur hypocrisie fondamentale, et maintenir, contre les propagandes, les chances que l'histoire peut avoir encore de devenir claire» (Humanisme et terreur, p. 298). Ce sont ces raisons de clarification de l'histoire et d'humanisme qui poussent Merleau-Ponty à l'extrême honnêteté de désavouer ses propres positions jugées fausses. Comme il l'indique dans sa correspondance avec Sartre («Sartre, Merleau-Ponty: les lettres d'une rupture», in Parcours II) il décide, après la guerre de Corée en 1950, de ne plus écrire sur les événements à mesure qu'ils se présentent, pour éviter l'engagement ponctuel sur chaque fait singulier, et les évaluer dans le tout d'une politique qui en change le sens. 
En résulte une conception de l'engagement distincte de celle de Sartre. Pour celui-ci, affirme Merleau-Ponty, s'engager n'est pas s'interpréter et se critiquer au contact de l'histoire, mais recréer soi-même sa relation avec elle et décider de tenir pour absolu le sens qu'on invente de donner à son histoire et à l'histoire publique. Pour Merleau-Ponty, au contraire, l'engagement consiste à ne pas définir par contrat ses rapports avec le dehors, à cesser de considérer le sens choisi pour sa propre vie comme source absolue, à relativiser ses propres critères, qui peuvent être infirmés par les épreuves de l'histoire. C'est cet engagement qui pousse Merleau-Ponty à choisir l'a-communisme : condition stricte de connaissance de l'URSS, car cette attitude confronte l'idéologie à la réalité, il est aussi condition d'une critique moderne du capitalisme. Il n'est donc plus possible de continuer à penser, comme il le faisait dans Humanisme et terreur, que si la société soviétique est loin des critères révolutionnaires du marxisme, la dialectique marxiste reste valable négativement, à titre de critique. C'est là, pour Merleau-Ponty, placer le marxisme hors des conditions de l'histoire, le justifier pour des raisons qui ne sont pas les siennes, dissocier critique et action marxiste alors qu'elles ne sont qu'un mouvement. C'est pourquoi le philosophe revient sur ses positions décrites dans Humanisme et terreur, les qualifie d'attentisme marxiste, qui dans la situation nouvelle devient action communiste. Contrairement à l'engagement sartrien qui confronte choses et consciences comme deux ordres opposés, l'engagement de Merleau-Ponty vise cet entre-deux des événements et des pures pensées, des choses et des esprits, celui des actions symboliques, auquel «appartiennent les livres, les conférences, mais aussi les meetings ». Le terrorisme d'État de l'URSS, oppression systématique de toute opposition et politique internationale belliqueuse, conduit donc le phénoménologue à rectifier ses positions, mais aussi à abandonner la philosophie même de l'histoire qui lui servait d'outil pour comprendre l'histoire et s'y positionner.

Dogmatisme d'une vérité absolue et d'un sens de l'histoire défini pour toujours, rigidification d'une idéologie qui entend non point s'accorder à la réalité mais la soumettre à son analyse par une série de rectifications toutes volontaristes, répression et violence contre tout ce qui s'y oppose, fuite en avant dans toujours plus d'ordre : aura-t-il fallu attendre l'analyse de Merleau-Ponty pour ainsi définir le terrorisme d'État? Certes, cette étude ne semble apporter rien de particulièrement nouveau dans sa caractérisation, mais la conclusion à laquelle on parvient est différente selon que l'analyse est effectuée ou non. Si le parcours de Merleau-Ponty aboutit davantage à une réflexion sur la position du philosophe, et partant, des sciences humaines devant de tels faits, il développe également certains points susceptibles d'être repris pour considérer le terrorisme actuel.

C'est d'abord l'importance de l'altérité, au centre des analyses de MerleauPonty, qui pose la question des modalités du rapport à autrui dans le terrorisme. On ne tue pas pour un progrès relatif, au nom de principes susceptibles de s'autocritiquer: à l'intersubjectivité s'oppose ici l'absolu que le terrorisme prend pour but. Dans le terrorisme d'État, l'absolu pour lequel agissait la terreur 
finit par devenir la conservation de l'appareil d'État, et le maintien de l'ordre. C'est là le paradoxe d'un régime qui, s'étant fixé l'humanisme pour absolu, en arrive à une négation totale de l'altérité. Il n'y a pas d'autre doublement: par dogme, car le parti est infaillible et n'accepte pas de dissension, mais aussi par violence, on élimine l'autre s'il persiste à ne pas s'aligner.

Il convient d'insister aussi, comme le fait Merleau-Ponty, sur le fondement humaniste à partir duquel s'est construit le communisme, de manière à ne pas le confondre avec le nazisme, sous l'appellation de «totalitarisme » contestée pour cette raison. Merleau-Ponty le voyait bien, en écrivant dans «L'URSS et les camps» que le fascisme est une angoisse devant le bolchevisme dont il reprend la forme extérieure pour en détruire le contenu de Stimmung internationaliste et prolétarienne. Le communisme est à entendre dans le cadre d'une philosophie de l'histoire qui connaît un échec quand cela même qu'elle revendique comme sa spécificité, la dialectique marxiste, se retourne en son contraire, le positivisme volontariste.

La position à laquelle Merleau-Ponty semble mener les sciences humaines consiste à maintenir un souci herméneutique d'interprétation de l'histoire, et à dénoncer les rigidifications d'absolus extra-historiques qui bloquent la compréhension.

À l'issu de ce parcours, nous sommes conduits à nous interroger sur la possibilité pour les sciences humaines d'appréhender le phénomène du terrorisme, tout en demandant conjointement si l'on peut reconnaître un terrorisme d'État actuellement. Il ne semble pas exister aujourd'hui de terrorisme d'État exerçant une répression aussi directement repérable et aboutissant jusqu'à l'absurde suprême du totalitarisme qui vise toujours plus d'ordre. Cependant, certains points peuvent être identifiés, moins dans les faits que dans l'ordre des discours. Remarquons d'abord l'affinité du terrorisme, ici dégagée, pour des abstractions comme la «science pure», ou, pour le régime communiste, «l'histoire universelle», «la vérité historique», «la société sans classes ». Ces termes tiennent lieu d'abstraction car ils sont extraits de la chair de leur surgissement - du fait de la rupture de la dialectique et du figement du sens conséquent et plaquent sur une réalité des schémas erronés non susceptibles d'être rectifiés par les faits. Ce sont des abstractions similaires qui peuvent être développées par un discours officiel des médias ou du pouvoir souhaitant appréhender des phénomènes terroristes aujourd'hui. Une certaine analyse reprenant la théorisation d'un Samuel Huttington dans son article «The Clash of Civilizations » (1993) ou dans son ouvrage éponyme (1996) théâtralise l'affrontement de deux identités culturelles, «civilisationnelles » pétrifiées : 1'Occident et l'Islam. On retrouve là un manichéisme comparable à celui que Merleau-Ponty dénonçait en renvoyant dos à dos communisme et monde libre. Pernicieusement, cette opposition tout entière construite sur des abstractions est reprise à leur compte par ceux qui perpètrent aujourd'hui des actes terroristes. Ce phénomène nouveau, fonctionnant à coup de généralisations planétaires, nous amène à redéfinir le partage traditionnel entre terrorisme d'organisations ou d'individus 
et terrorisme d'État. Surgit alors un terrorisme qui reprend au terrorisme d'organisations sa dispersion, la violence de sa terreur, la spectacularité de ses exactions, et au terrorisme d'État son pouvoir établi - à une échelle non plus étatique mais internationale -, un terrorisme des blocs, blocage de dogmes simplistes mis en scènes puis récupérés par ceux qui tuent pour un absolu «Jihad» ou autre - et ceux qui prétendent s'y opposer pour une autre «Croisade du bien contre le mal», ou «Justice infinie».

La tâche des sciences humaines est alors de lever l'ignorance, d'affiner l'étude, d'effectuer une compréhension critique des détails, d'empêcher la pétrification du sens dans des abstractions, de révéler la démagogie des analyses manichéennes, en un mot, de retrouver derrière les conflits culturels mis en scènes le fondement, proximité et différence, de l'altérité.

Thamy AYOUCH

Doctorant, Assistant Moniteur Normalien École Doctorale «Recherches en Psychanalyse»

Université Paris 7-Denis Diderot

20 rue St Sauveur 75002 Paris

\section{BIBLIOGRAPHIE}

ARENDT H. Le Système totalitaire (Les Origines du totalitarisme, tome 3), Paris, Seuil, 1972.

DUPOND P. Le Vocabulaire de Merleau-Ponty, Paris, Ellipses, 2001.

GADAMER H.G. Vérité et méthode, Paris, Seuil, 1996.

HEGEL G.W.F. Phénoménologie de l'Esprit, (trad. Jean-Pierre Lefebvre), Paris, Aubier, 1991.

MARX K., «Argent, État, Prolétariat », in Philosophie, Paris, Gallimard, 1965, 1968, 1982, pp. 19-134.

MARX K., «Économie et Philosophie (Manuscrits parisiens)», in Philosophie, Paris, Gallimard, 1965, 1968, 1982, pp. 137-222.

MARX K., «L'idéologie allemande «Conception matérialiste et critique du monde »», in Philosophie, Paris, Gallimard, 1965, 1968, 1982, pp. 289-392.

MARX K., «Le manifeste communiste», in Philosophie, Paris, Gallimard, 1965, 1968, 1982, pp. 395-440.

MERLEAU-PONTY M. La Structure du comportement, Paris, P.U.F., 1942.

MERLEAU-PONTY M. Phénoménologie de la perception, Paris, Gallimard, 1945.

MERLEAU-PONTY M. Humanisme et terreur, Paris, Gallimard, 1947.

MERLEAU-PONTY M. «Le philosophe et la sociologie», in Éloge de la philosophie et autres essais, Paris, Gallimard, 1953, pp. 97-122. 
MERLEAU-PONTY M. «Partout et nulle part», in Éloge de la philosophie et autres essais, Paris, Gallimard, 1953, pp. 143-198.

MERLEAU-PONTY M. «Note sur Machiavel», in Éloge de la philosophie et autres essais, Paris, Gallimard, 1953, pp. 287-308.

MERLEAU-PONTY M. Les Aventures de la dialectique, Paris, Gallimard, 1955.

MERLEAU-PONTY M. «La politique paranoïaque », in Signes, Paris, Gallimard, 1960, pp. 397-422.

MERLEAU-PONTY M. «Marxisme et superstition», in Signes, Paris, Gallimard, 1960, pp. 422-424.

MERLEAU-PONTY M. «L'URSS et les camps», in Signes, Paris, Gallimard, 1960, pp. 424-442.

MERLEAU-PONTY M. «Les papiers de Yalta », in Signes, Paris, Gallimard, 1960, pp. 442447.

MERLEAU-PONTY M. «L'avenir de la Révolution», in Signes, Paris, Gallimard, 1960, pp. 448-471.

MERLEAU-PONTY M. «Sur la déstalinisation », in Signes, Paris, Gallimard, 1960, pp. 472497.

MERLEAU-PONTY M. Le Visible et l'invisible, Paris, Gallimard, 1960.

MERLEAU-PONTY M. Le primat de la perception et ses conséquences philosophiques, Grenoble, Cynara, 1989.

MERLEAU-PONTY M. «L'existentialisme chez Hegel », in Sens et non-sens, Paris, Gallimard, 1996, pp 79-87.

MERLEAU-PONTY M. «La querelle de l'existentialisme», in Sens et non-sens, Paris, Gallimard, 1996, pp. 88-101.

MERLEAU-PONTY M. «Autour du marxisme», in Sens et non-sens, Paris, Gallimard, 1996, pp. 120-151.

MERLEAU-PONTY M. «Marxisme et philosophie », in Sens et non-sens, Paris, Gallimard, 1996, pp. 152-166.

MERLEAU-PONTY M. «Le manifeste communiste a cent ans », in Parcours 1935-1951, Paris, Verdier, 1997, pp. 103-108.

MERLEAU-PONTY M. «Sartre, Merleau-Ponty : les lettres d'une rupture », in Parcours II 1951-1961, Paris, Verdier, 2000, pp. 129-169.

MERLEAU-PONTY M. «L'avenir du socialisme», in Parcours II 1951-1961, Paris, Verdier, 2000, pp. 241-246.

MERLEAU-PONTY M. «La philosophie et la politique sont solidaires », in Parcours II 1951-1961, Paris, Verdier, 2000, pp. 302-304.

SARTRE J.P., L'Être et le Néant, Paris, Gallimard, 1943s. 
Thamy Ayouch - De la terreur au terrorisme: les aventures d'une philosophie de l'histoire chez Merleau-Ponty

Résumé : En suivant Merleau-Ponty dans ses ouvrages Humanisme et Terreur, Les Aventures de la dialectique, et dans des articles traitant du marxisme, nous nous proposons de souligner comment le constat d'un terrorisme d'État soviétique conduit à réviser les outils théoriques et pratiques dont peut disposer le philosophe pour comprendre l'histoire et s'y positionner. Lorsque la terreur, inséparable d'une finalité placée hors d'elle, l'humanisme, est reconnue comme terrorisme d'État, qui finit par ne plus viser que lui-même, on aboutit à une remise en question de la portée herméneutique de la dialectique marxiste. Ce figement du sens dans des absolus extra-historique est ce qui caractérise également le «terrorisme de blocs» actuel, rapport spéculaire entre médias et acteurs violents.

Mots-clés : Terrorisme d'État - Dialectique - Herméneutique - Engagement.

Thamy Ayouch - From Terror to Terrorism - The Adventures of a Philosophy of History as defined by Merleau-Ponty

Summary : Our reading of Merleau-Ponty in works such as Humanism and Terror or The Adventures of Dialectics and in articles on Marxism, helps us to demonstrate how the recognition of the terrorism organised by the Soviet State implies the philosopher revising the theoretical and practical tools at his disposal in the understanding of history and also enables him to establish his own stance. When terror, the finality of which is humanism, an external force, becomes State terrorism, whose goal must be only itself, the hermeneutic reach of Marxist dialectics is then questioned. This petrifaction of meaning in extra-historical ideals is also what characterises the present-day 'blocks terrorism,' a mirror relation between the media and violent perpetrators.

Key-words : State-organised Terrorism - Dialectics - Hermeneutics - Commitment. 